\title{
Scapular dyskinesis increases the risk of future shoulder pain by $43 \%$ in asymptomatic athletes: a systematic review and meta-analysis
}

\author{
Darren Hickey, Veronica Solvig, Vinicius Cavalheri, Meg Harrold, Leanda Mckenna
}

- Additional material is published online only. To view, please visit the journal online (http://dx.doi.org/10.1136/ bjsports-2017-097559).

School of Physiotherapy and Exercise Science, Faculty of Health Sciences, Curtin University, Perth, Western Australia, Australia

\section{Correspondence to} Dr Leanda Mckenna, School of Physiotherapy and Exercise Science, Faculty of Health Science, Curtin University, Kent St, Bentley WA 6102, Australia I.mckenna@curtin.edu.au

Accepted 28 May 2017 Published Online First 22 July 2017

\section{SLinked}

http://dx.doi.org/10.1136/ bjsports-2017-098233

\section{CrossMark}

\section{To cite: Hickey $D$,}

Solvig V, Cavalheri V,

et al. Br J Sports Med

2018:52:1-10.

\section{ABSTRACT}

Background It is unclear whether the presence of scapular dyskinesis increases the risk of developing shoulder pain in asymptomatic athletes.

Objectives To determine whether the presence of scapular dyskinesis in asymptomatic athletes increases the risk of developing shoulder pain by systematic review and meta-analysis.

Methods A systematic search was conducted in the Cochrane Library, Embase, PubMed, Cumulative Index to Nursing and Allied Health Literature, Allied and Complementary Medicine Database and SPORTDiscus. Prospective studies that assessed athletes for scapular dyskinesis and recorded incidents of shoulder pain were included. Study quality was assessed using the Downs and Black checklist. Meta-analysis was conducted to derive a pooled risk ratio (RR) for the development of shoulder pain in athletes with scapular dyskinesis compared with those without scapular dyskinesis.

Results Five studies were included with a total of 419 athletes. Of the athletes with scapular dyskinesis, $35 \%(56 / 160)$ experienced shoulder pain during the follow-up, whereas $25 \%$ (65/259) of athletes without scapular dyskinesis experienced symptoms. The presence of scapular dyskinesis at baseline indicated a 43\% increased risk of a shoulder pain event over a 9 to 24 months follow-up (RR=1.43, 95\% Cl 1.05 to 1.93 ).

Conclusions Athletes with scapular dyskinesis have $43 \%$ greater risk of developing shoulder pain than those without scapular dyskinesis.

\section{INTRODUCTION}

Racket and overhead sports require substantial kinetic energy transference through the shoulder at rapid speeds through large ranges of motion with high precision. ${ }^{12}$ These demands may explain the high prevalence of shoulder pain in these populations, with reports of $12 \%$ in amateur golf, ${ }^{3} 16 \%$ in volleyball, ${ }^{4} 22 \%$ to $36 \% \%$ in elite handball ${ }^{45}$ and $24 \%$ in high-level adolescent tennis, which increases to $50 \%$ in middle-aged tennis players. ${ }^{6}$ Even higher is the prevalence of shoulder pain in swimmers, ranging between $40 \%$ and $91 \% .^{7}$ In collision sports such as rugby and American football, 6.7\% and $15.2 \%$ of all injuries involve the shoulder. ${ }^{8}$ These collision sport statistics are not inclusive of non-traumatic sources of shoulder pain, and may under-represent the true prevalence of all shoulder pain in these populations. ${ }^{10}$

The traditional approach to understanding the mechanisms of shoulder pain has involved specific anatomical diagnostic labels. The validity of using specific anatomical diagnostic labels has been challenged extensively, particularly regarding non-acute shoulder pain. ${ }^{11-13}$ Clinical features of specific anatomical diagnoses, such as reduced external rotation or an external rotation lag, indicative of frozen shoulder and rotator cuff tear, respectively, are inconsistent predictors of outcome. ${ }^{14}$ Specific anatomical diagnostic labels provide limited clinical guidance in patient management or estimating prognosis. ${ }^{15}$ For these reasons, experts are calling for a paradigm shift away from these labels and encouraging identifying modifiable risk factors associated with onset of shoulder pain or that influence prognosis, such as abnormal motor patterns or movement impairments. ${ }^{16}$

One potential risk factor for shoulder pain is scapular dyskinesis. Scapular dyskinesis refers to altered position and motion of the scapula. ${ }^{1}$ Scapular dyskinesis may reduce subacromial space, ${ }^{17}$ although the evidence for this is mixed. ${ }^{18}$ Scapular dyskinesis can also reduce rotator cuff strength, ${ }^{19} 20$ increase the strain within the rotator cuff and promote apoptotic changes in tenocytes within the rotator cuff tendons. ${ }^{1}$ Rotator cuff weakness may impair motor control, resulting in superior translation of the humeral head and further mechanical abrasion of the structures of the subacromial space. ${ }^{21}$ Despite these plausible mechanisms that may cause shoulder pain, clinical evidence to support these hypotheses remains limited.

Scapular dyskinesis has been associated with shoulder pain, specifically shoulder impingement syndrome (SIS), rotator cuff tendinopathy and multidirectional impairments. ${ }^{1} 22$ The cross-sectional nature of the studies in previous reviews means one cannot determine whether scapular dyskinesis contributed to the development of shoulder pain or whether it arose subsequent to shoulder pain. Scapular dyskinesis is highly prevalent in the asymptomatic general population ${ }^{23}$ and substantially higher in overhead athletes. ${ }^{23}$ It remains unclear if dyskinesis is a sports-specific adoption that is potentially beneficial for maximal performance and protective against injury. Alternatively, it may be a risk factor that identifies athletes at greater risk of injury, requiring a preventative intervention. Two prospective studies attempted to identify whether scapular dyskinesis in asymptomatic athletes increased their risk of developing shoulder pain and results were mixed. ${ }^{24} 25$ Therefore, we aimed to systematically review whether the presence of scapular dyskinesis in asymptomatic athletes increased the risk of developing future shoulder pain. 


\begin{tabular}{|c|c|}
\hline Inclusion criteria & Exclusion criteria \\
\hline Prospective observational studies & $\begin{array}{l}\text { Randomised control trials, cross-sectional } \\
\text { studies and other }\end{array}$ \\
\hline Athletic population & Pain at baseline \\
\hline Pain-free participants at baseline & Non-musculoskeletal aetiology \\
\hline Valid baseline assessment of dyskinesis* & $\begin{array}{l}\text { Non-shoulder-related musculoskeletal } \\
\text { pathologies }\end{array}$ \\
\hline $\begin{array}{l}\text { Assessment of self-reported shoulder } \\
\text { pain at follow-upt }\end{array}$ & $\begin{array}{l}\text { Inability to obtain dichotomised data } \\
\text { on the presence of dyskinesis or } \\
\text { development of shoulder pain }\end{array}$ \\
\hline \multicolumn{2}{|l|}{$\begin{array}{l}\text { English, Norwegian, Swedish, Danish, } \\
\text { Portuguese and Spanish }\end{array}$} \\
\hline
\end{tabular}

\section{METHODS}

\section{Search strategy}

This review has been registered in the PROSPERO database (CRD42016046247) and conducted according to the Preferred Reporting Items for Systematic reviews and Meta-Analyses guidelines. $^{26}$

The following databases were used for searching existing literature (from their inception to August 2016): Cochrane Library, Embase (via Ovid), PubMed, Cumulative Index to Nursing and Allied Health Literature (via Elton B Stephens Co (EBSCO)), Allied and Complementary Medicine Database (via Ovid) and SPORTDiscus (via EBSCO). The full Embase search strategy is outlined in online supplementary appendix 1 and was adapted for the other databases. The reference lists of the included studies were checked for additional studies that were not identified with the database search. To identify recent research that was not yet published, a hand search was undertaken of abstracts presented at conferences (last 3 years) of; the Australian Physiotherapy Association, Sports Medicine Australia and American College of Sports Medicine.

\section{Study selection}

The search was conducted by two authors (DH, VS). Articles were exported to EndNote, ${ }^{27}$ and duplicates removed. The remaining articles were exported into Covidence, ${ }^{28}$ where the same two authors independently screened titles and abstracts to determine their eligibility based on the criteria outlined in table 1. Once agreement was reached, the full text of each article was reviewed to determine their inclusion or exclusion. Disagreements between the review authors (DH, VS) were resolved by discussion with a third review author (LM).

\section{Data extraction}

Two authors (DH, VS) independently extracted data of the included studies using a standardised form. Disagreements were resolved through discussion with a third review author (LM). Participants were classified according to whether scapular dyskinesis was observed at the initial assessment, and whether they had shoulder pain during the follow-up. Where only continuous data for measurements of scapular position were present, the authors were contacted to investigate if the data could be dichotomised, that is, categorised into with or without scapular dyskinesis. Where this was not possible, the studies were excluded. Authors were also contacted where there were missing data or where only partial data met the inclusion criteria, for example if some of the included population had shoulder pain at baseline or were non-athletic. The characteristics of the included studies, such as number of participants, patient characteristics and sporting participation, outcomes, findings and major strengths and weaknesses, were extracted.

\section{Quality assessment}

The included studies were assessed for methodological quality using the Downs and Black Checklist. ${ }^{29}$ The Downs and Black Checklist is recommended in the Cochrane Handbook for appraisal of non-randomised studies. ${ }^{30}$ It contains 27 yes/ no questions, with a total maximum score of 30 . The score is distributed over five sections: Study quality, external validity, study bias, confounding and selection bias and power of the study. Question 4, 8, 14, 19, 23, 24 and 27 were not applicable to prospective study designs, and were therefore excluded. This was based on the recommendations of a previous systemic review investigating risk factors using prospective study designs. ${ }^{31}$ The modified checklist had a maximum score of 20 .

\section{Outcome measures}

The main outcome of this review was shoulder pain recorded via any questionnaire, scale or tool that detected the dichotomised outcome of 'shoulder pain' or 'no shoulder pain' during the follow-up period. The outcome measures identified as acceptable measures of shoulder pain were the Visual Analogue Scale, the Numerical Rating Scale, the Shoulder Pain and Disability Index, the Disabilities of the Arm, Shoulder and Hand, Shoulder Disability Questionnaire, direct questioning, direct report to medical professionals and any other valid outcome measure of shoulder disability and/or pain. Visual observation, 3-D analysis of scapular position or use of any other measurement tool to determine the presence of scapular dyskinesis was accepted, as long as the data could be dichotomised as 'scapular dyskinesis' or 'no scapular dyskinesis'.

\section{Heterogeneity assessment}

Clinical and methodological heterogeneity across all included studies was examined and reported. Methodological heterogeneity was reported in a summary table. Statistical heterogeneity across studies was assessed by visual analysis of forest plots and the $\mathrm{I}^{2}$ test. The $\mathrm{I}^{2}$ test assessed the percentage of inconsistency (ie, variability) due to heterogeneity, and values greater than $50 \%$ were considered substantial heterogeneity. ${ }^{30}$

\section{Data analysis}

Review Manager (RevMan) V.5.3 was used to perform statistical analyses and to generate forest plots (Copenhagen: The Nordic Cochrane Centre, The Cochrane Collaboration, 2014). ${ }^{32}$ The primary outcome measure was shoulder pain, and the pooled risk ratio (RR) and 95\% CI were calculated. A random effects model was applied if substantial heterogeneity was found, otherwise, a fixed effects model was used. The meta-analysis was performed using the Mantel-Haenszel method. ${ }^{33}$ Sensitivity analyses were undertaken to determine if the outcome was affected by different classification methods from individual studies.

\section{RESULTS}

\section{Study selection}

The full search strategy and selection process are outlined in figure 1 . The initial database search was completed on 9 August 2016, with a hand search of conference abstracts conducted the following day. This search identified 1099 study titles that potentially met inclusion/exclusion criteria. After duplicates 


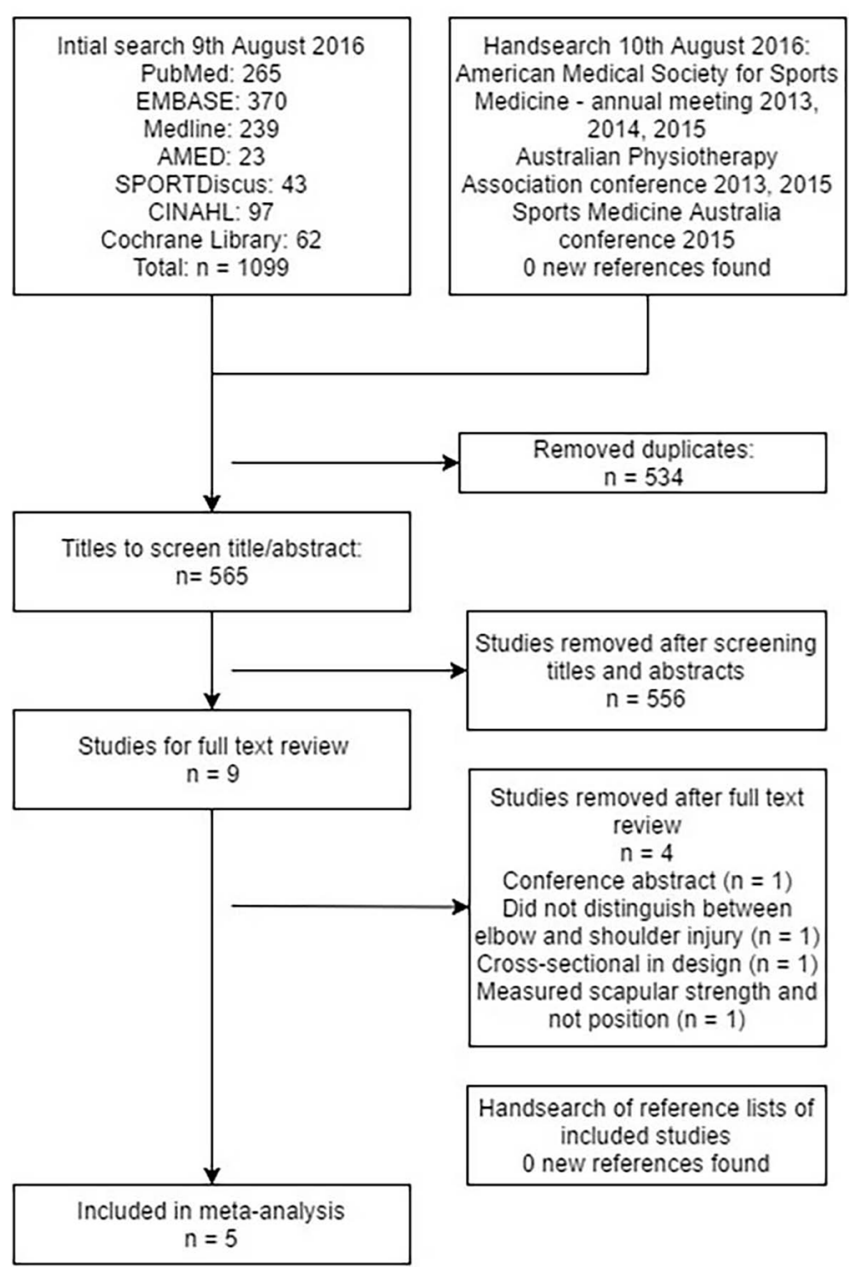

Figure 1 Flow chart of included studies. AMED, Allied and Complementary Medicine Database; CINAHL, Cumulative Index to Nursing and Allied Health Literature.

were removed, the remaining 565 studies were screened, with nine studies reviewed in full text. After successfully contacting three authors, ${ }^{24} 3435$ five studies met the inclusion and exclusion criteria and were included in the meta-analysis. ${ }^{36} 37$ Of the four studies excluded at full-text review, one text was not available in full text, ${ }^{38}$ one was cross-sectional in study design, ${ }^{39}$ one did not distinguish between shoulder and elbow injuries $^{25}$ and one measured scapular muscle strength but not scapular dyskinesis. ${ }^{40}$

\section{Study characteristics}

Table 2 outlines the characteristics of the five included studies. A total of 419 participants were included in the meta-analysis, with the mean age ranging from 14 to 34 . The cohorts used by the included studies ranged from recreational to elite athletes. Two cohorts consisted of adolescents, ${ }^{34} 35$ while the remaining studies included adults. ${ }^{24} 3637$ Studies predominantly investigated athletes participating in overhead sports, from swimming to throwing and racket sports, ${ }^{24} 343537$ with one investigating a population of rugby players. ${ }^{36}$ Two studies included participants with self-reported shoulder pain at baseline, and these participants were excluded from the analysis. ${ }^{24} 34$

Four of the five studies used different variations of visual dynamic assessment. ${ }^{24} 343637$ The visual dynamic qualitative assessment method was initially described by Kibler et $a l^{41}$ using a four-part classification, which utilised three different types of scapular dyskinesis, with a fourth classification representing cases without scapular dyskinesis. ${ }^{41}$ More recently, this method has been simplified to a dichotomous classification(with or without scapular dyskinesis) ${ }^{42}$ or an obvious/subtle/no scapular dyskinesis trichotomous classification ${ }^{43} 44$ or a winging/ tilting/normal trichotomous classification. ${ }^{45}$ Where a study used multiple classifications, this was dichotomised into with or without scapular dyskinesis for meta-analysis. ${ }^{24} 3637$ Only one study differed substantially from these methods of visual classification, using measurement of distance between anatomical landmarks. ${ }^{35}$ The author of that study provided dichotomised data (with or without scapular dyskinesis) using receiver operating characteristic analysis. This process is outlined in detail in online supplementary appendix 2 .

In the included studies, participants were followed up for either 1 year or one season. However, one study performed follow-up at 12 and 24 months. ${ }^{37}$ All studies utilised self-reporting of shoulder pain/injuries. A single end of season questionnaire was used in three studies. ${ }^{34-36}$ One of these studies also used self-report at medical check-ups. ${ }^{34}$ Serial administration of standardised questionnaires was used in two studies. The Shoulder Disability Questionnaire was administered at 12 and 24 months by Struyf et al. ${ }^{37}$ The Oslo Sports Trauma Research Centre injury questionnaire was administered every 2 weeks throughout the season by Clarsen et al. ${ }^{24}$

Two studies classified shoulder pain as reporting pain at any point during the follow-up, lasting at least a day. ${ }^{35} 37$ One study classified shoulder pain as pain lasting at least 2 weeks, but not necessarily requiring withdrawal from sport. ${ }^{36}$ One study classified shoulder pain as requiring greater than 7 days of withdrawal from participation. ${ }^{34}$ One study administered 15 questionnaires and an average score called the Cumulative Severity Score (CSS) was calculated. ${ }^{24}$ In their analysis they used a cut-off of $>40 \%$ to define shoulder injury. This cut-off was used because it had the greatest predictive value. For the purpose of this study, a cut-off point of a CSS $>0$ was used. This would detect the presence of any shoulder pain during the follow-up and increase similarity with the other studies. The total prevalence of shoulder pain was $121 / 419$ (26.95\%, range $18.2 \%$ to $40.3 \%)$.

\section{Risk of bias}

The critical appraisal of the included studies using the Downs and Black checklist is detailed in figure 2 and summarised using a stacked bar chart in figure 3. Generally, studies performed well, with four obtaining greater than $75 \%$ scores and a mean score of $81 \% .^{2434-36}$ However, some risks of bias were common. No study reported the results of the baseline assessment of scapular dyskinesis of drop-outs, and it cannot be determined if scapular dyskinesis was equally prevalent in those who dropped out and those that did not. No study made between-group comparisons between those with and without scapular dyskinesis at baseline. Only two studies compared other risk factors between cases and controls to further explain their findings. ${ }^{34} 35$ Only one study fully met the external validity criteria by clearly outlining their recruitment process. ${ }^{24}$ By not reporting the proportion of the source population from which the patients were derived and proportion of the sample who were included, it could not be accurately established whether the cohorts were representative of the source population in the other four studies. ${ }^{34-37}$ Outside of the checklist, no study reported if participants were blinded to their baseline assessment of scapular dyskinesis. 


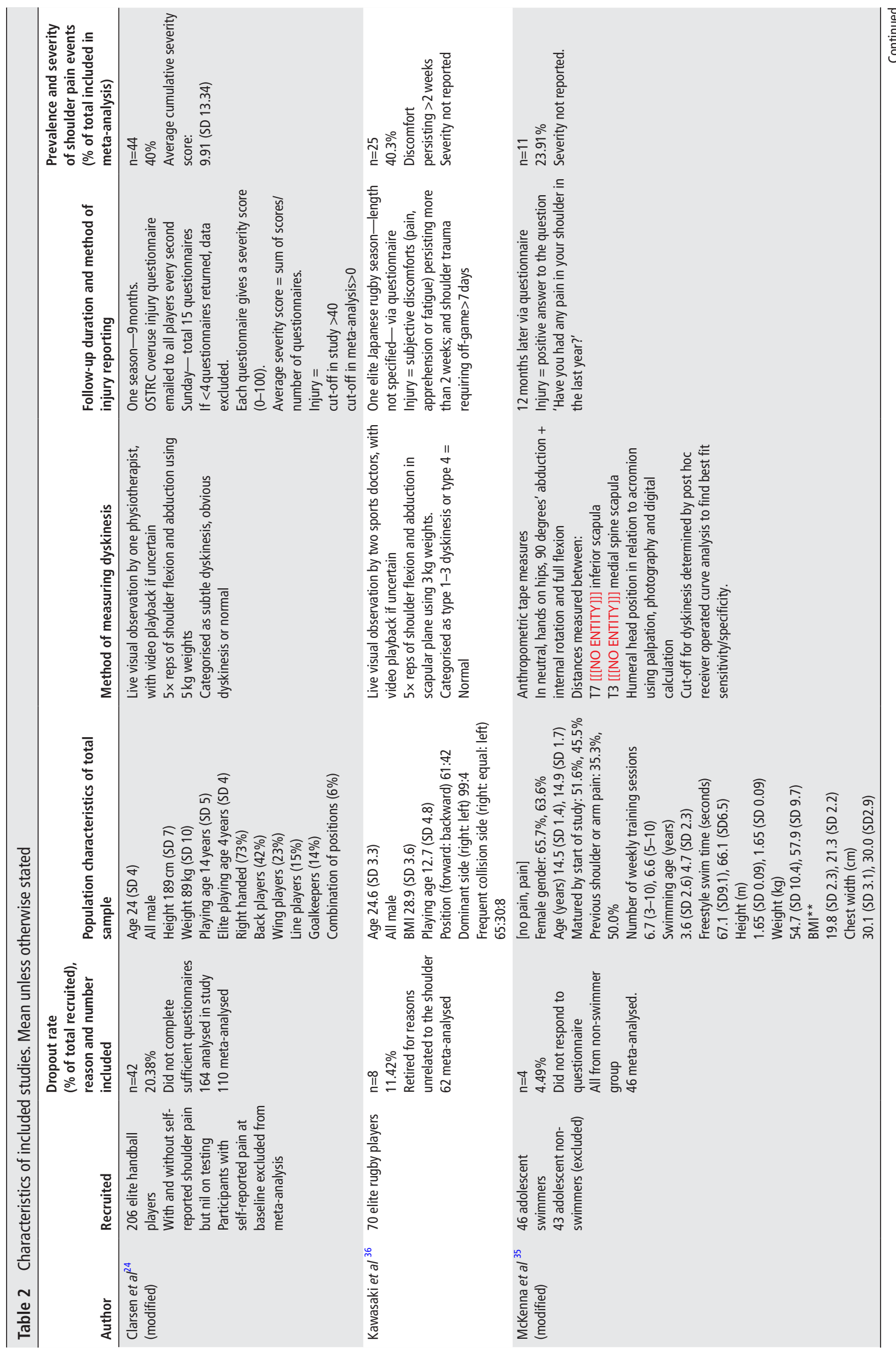




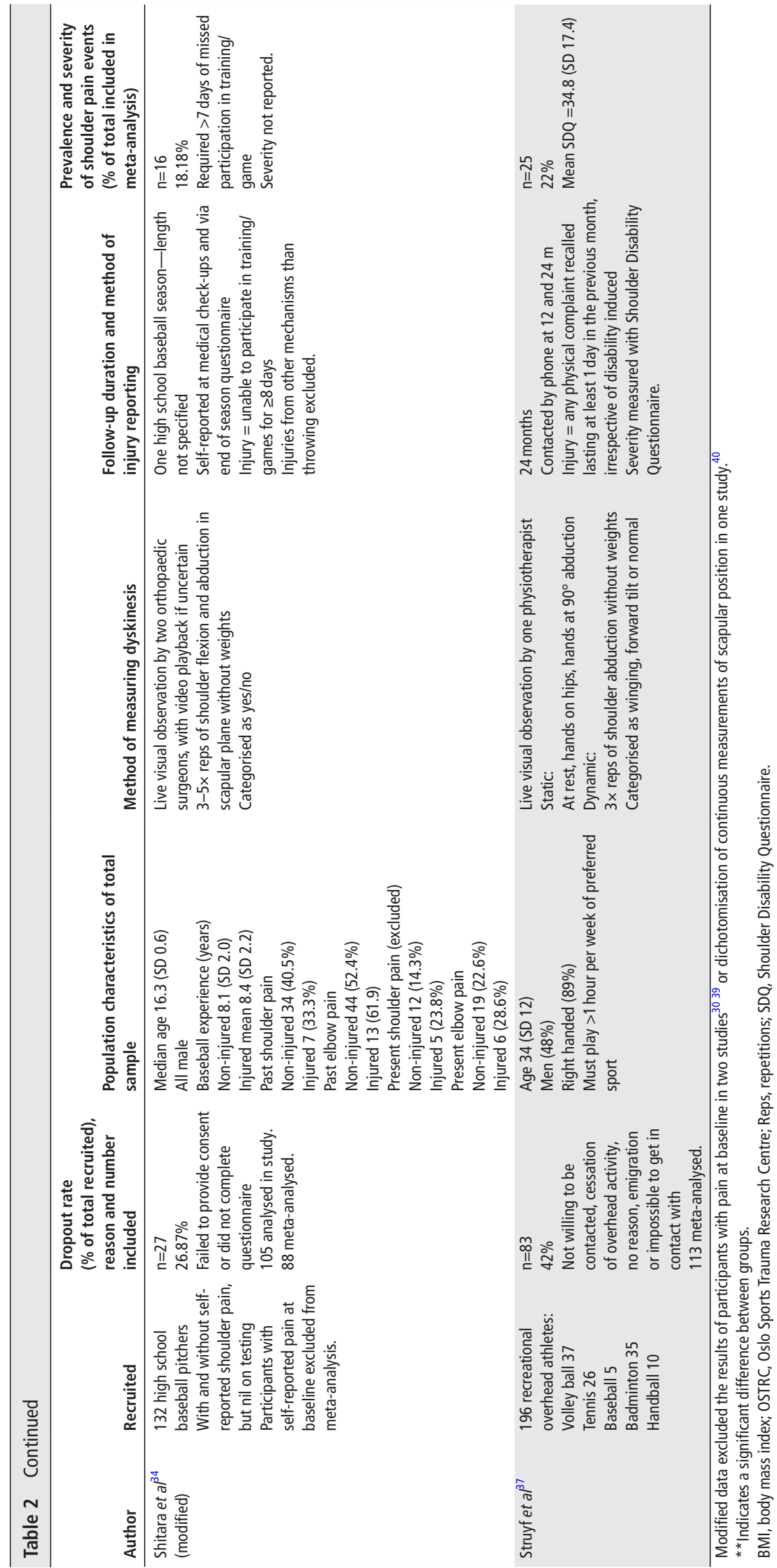

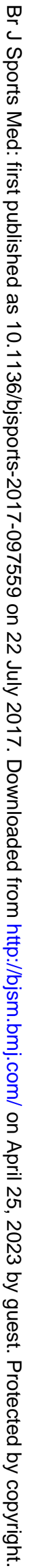




\begin{tabular}{|c|c|c|c|c|c|c|c|c|c|c|c|c|c|c|c|c|c|c|c|c|c|c|c|c|c|c|c|c|c|}
\hline & 1 & 2 & 3 & 4 & 5 & 6 & 7 & 8 & 9 & 10 & 11 & 12 & 13 & 14 & 15 & 16 & 17 & 18 & 19 & 20 & 21 & 22 & 23 & 24 & 25 & 262 & 27 & Score (Total=20) & Percent \\
\hline Clarsen & Y & Y & Y & $x$ & $\mathrm{~N}$ & Y & Y & $x$ & $\mathrm{~N}$ & Y & Y & Y & Y & $x$ & Y & Y & Y & Y & $x$ & Y & $Y$ & Y & $x$ & $x$ & $\mathrm{~N}$ & $\mathrm{~N}$ & $x$ & 16 & 80 \\
\hline Kawasaki & Y & Y & Y & $x$ & $\mathrm{~N}$ & Y & Y & $x$ & Y & $Y$ & $Y$ & $\mathrm{~N}$ & Y & $x$ & $Y$ & Y & Y & Y & $x$ & Y & Y & Y & $x$ & $x$ & $\mathrm{~N}$ & Y & $x$ & 17 & 85 \\
\hline McKenna & Y & Y & Y & $x$ & $Y$ & Y & Y & $x$ & $Y$ & $Y$ & $Y$ & $\mathrm{~N}$ & $Y$ & $x$ & $Y$ & $Y$ & $Y$ & $Y$ & $x$ & $Y$ & Y & $Y$ & $x$ & $x$ & Y & Y & $x$ & 19 & 95 \\
\hline Shitara & $Y$ & Y & Y & $x$ & $Y$ & $Y$ & Y & $x$ & $\mathrm{~N}$ & $Y$ & $Y$ & $\mathrm{~N}$ & Y & $x$ & $Y$ & $Y$ & Y & Y & $x$ & Y & $Y$ & $Y$ & $x$ & $x$ & $\mathrm{~N}$ & $\mathrm{~N}$ & $x$ & 16 & 80 \\
\hline Struyf & $Y$ & $Y$ & Y & $x$ & $\mathrm{~N}$ & Y & $Y$ & $x$ & $\mathrm{~N}$ & $Y$ & $\mathrm{~N}$ & $N$ & $Y$ & $x$ & $\mathrm{~N}$ & $Y$ & $Y$ & $Y$ & $x$ & $Y$ & Y & Y & $x$ & $x$ & $\mathrm{~N}$ & $\mathrm{~N}$ & $x$ & 13 & 65 \\
\hline
\end{tabular}

Figure 2 Critical appraisal of the included studies using a modified Downs and Black checklist.

\section{Synthesis of results and meta-analysis}

All five studies were eligible to be included in the meta-analysis, of which the results are presented in figure 4 . Of the 419 participants, 160 (38.19\%) presented with scapular dyskinesis. In relation to the overall risk, $35 \%$ of athletes with scapular dyskinesis $(56 / 160)$ experienced shoulder pain over a 9 to 24 -month follow-up. In comparison, $25 \%$ of athletes without scapular dyskinesis (65/259) experienced shoulder pain in this time period.

The presence of scapular dyskinesis at baseline was indicative of a $43 \%$ increased risk of shoulder pain over a 9 to 24 -month follow-up $\left(\mathrm{RR}=1.43,95 \% \mathrm{CI} 1.05\right.$ to $\left.1.93, \mathrm{I}^{2}=40 \%\right)$. To determine the effects of a different classification of shoulder pain, Shitara $e t a l^{34}$ was excluded. The results of this sensitivity analysis demonstrated that the presence of scapular dyskinesis at baseline was indicative of a $54 \%$ increased risk of shoulder pain $\left(\mathrm{RR}=1.54,95 \% \mathrm{CI} 1.13\right.$ to $\left.2.10, \mathrm{I}^{2}=40 \%\right)$. To determine the effects of a different assessment of scapular dyskinesis, McKenna et $a l^{35}$ was excluded. The results of this sensitivity analysis demonstrated that the presence of scapular dyskinesis at baseline was indicative of a $28 \%$ increased risk of shoulder pain $\left(\mathrm{RR}=1.28\right.$, CI 0.93 to $\left.1.76, \mathrm{I}^{2}=17 \%\right)$. However, this result did not reach statistical significance.

\section{DISCUSSION}

This review extends knowledge of the linkage between scapular motion and pain, and found that asymptomatic athletes with scapular dyskinesis have a $43 \%$ greater risk of developing shoulder pain over a 9 to 24-month follow-up period compared with counterparts who did not have scapular dyskinesis. This adds weight to the argument that scapular dyskinesis may contribute to the development of shoulder pain. Scapular dyskinesis might be considered a 'culprit' rather than a 'victim' in some cases of shoulder pain. However, these results should be interpreted with caution due to the variance in the 95\% CIs, methodological heterogeneity and some risk of bias across the included studies.

\section{Analysis of key factors-classification of shoulder pain and measurement of scapular dyskinesis}

Both the classification of shoulder pain and the measurement of scapular dyskinesis varied across the included studies. Regarding shoulder pain, the classification used by Shitara $e t a l^{34}$ included a complete withdrawal from participation in training or competition of greater than 7 days; a stricter definition than those used by the other included studies. ${ }^{2435-37}$ Sensitivity analysis excluding the study by Shitara $e t a l^{34}$ increased the RR and narrowed the 95\% CI for increased risk of shoulder pain in asymptomatic athletes with scapular dyskinesis (RR $1.54,95 \%$ CI 1.12 to 2.10 ). This analysis suggests that scapular dyskinesis may not be a risk factor for injuries that require complete withdrawal from shortterm participation, that is, severe and disabling injuries. It may be a more important risk factor for lower grade shoulder injuries. Lower grade injuries still cause athletes to seek treatment and frequently cause reductions in performance and participation without being severe enough to cause complete withdrawal from sport. ${ }^{4}$

Measurement of scapular dyskinesis varied, as one included study $^{35}$ reported scapular dyskinesis using continuous data,

\section{Quality Appraisal}

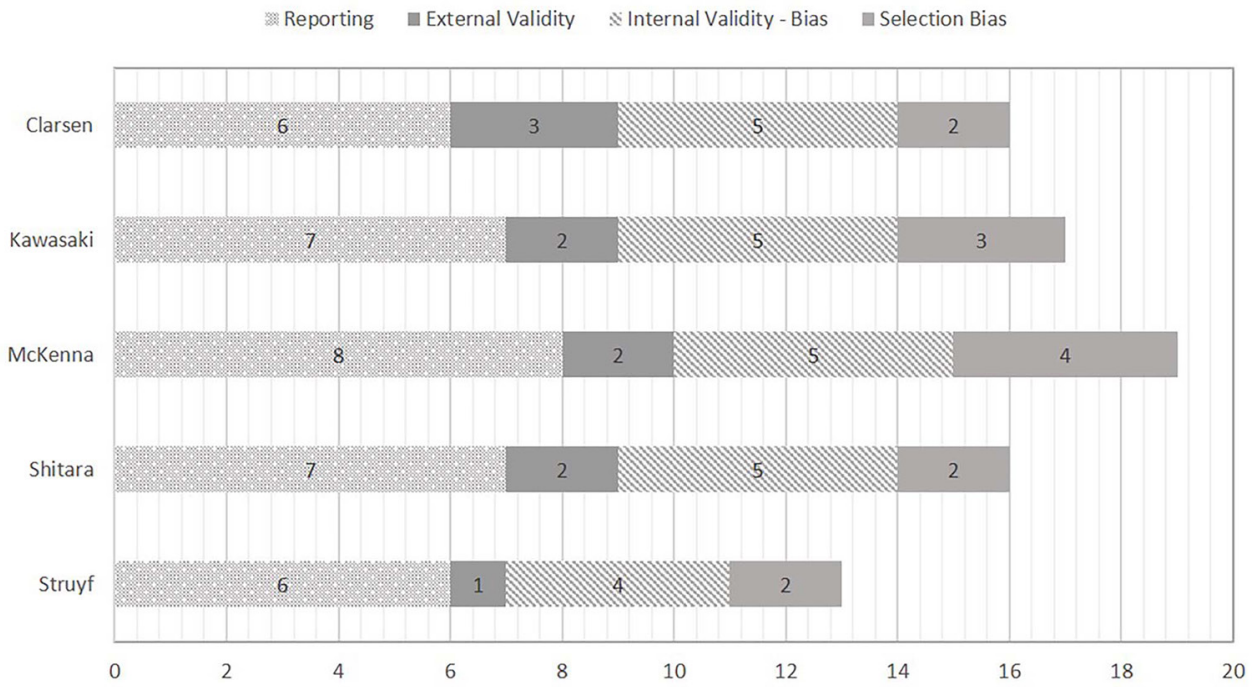

Figure 3 Stacked bar chart of study quality according to the modified Downs and Black checklist. 


\begin{tabular}{|c|c|c|c|c|c|c|c|c|c|c|}
\hline \multirow[b]{2}{*}{ Study or Subgroup } & \multicolumn{2}{|c|}{ Dyskinesis } & \multicolumn{2}{|c|}{ No dyskinesis } & \multicolumn{3}{|c|}{ Risk Ratio } & \multirow{2}{*}{\multicolumn{2}{|c|}{$\begin{array}{c}\text { Risk Ratio } \\
\mathrm{M}-\mathrm{H}, \text { Fixed, } 95 \% \mathrm{Cl}\end{array}$}} & \\
\hline & Events & Total & Events & Total & Weight & M-H, Fixed, $95 \% \mathrm{Cl}$ & & & & \\
\hline Clarsen 2014 & 21 & 49 & 23 & 61 & $45.2 \%$ & $1.14[0.72,1.79]$ & & & & \\
\hline Kawasaki 2012 & 9 & 14 & 16 & 48 & $15.9 \%$ & $1.93[1.10,3.37]$ & & & & \\
\hline McKenna 2012 & 8 & 18 & 3 & 28 & $5.2 \%$ & $4.15[1.27,13.60]$ & & & & \\
\hline Shitara 2015 & 2 & 17 & 14 & 71 & $11.9 \%$ & $0.60[0.15,2.38]$ & & & & \\
\hline Struyf 2014 & 16 & 62 & 9 & 51 & $21.8 \%$ & $1.46[0.71,3.03]$ & & & & \\
\hline Total $(95 \% \mathrm{Cl})$ & & 160 & & 259 & $100.0 \%$ & $1.43[1.05,1.93]$ & & & & \\
\hline Total events & 56 & & 65 & & & & & & & \\
\hline $\begin{array}{l}\text { Heterogeneity. } \mathrm{Chi}^{2}= \\
\text { Test for overall effect }\end{array}$ & $\begin{array}{l}6.70, d f= \\
Z=2.28(\end{array}$ & $\begin{array}{l}4\langle P=0 \\
P=0.0\end{array}$ & $\begin{array}{l}0.15) ; i^{2}= \\
\text { 2) }\end{array}$ & & & & 0.05 & $\begin{array}{l}0.2 \\
\text { No dyskinesis }\end{array}$ & Dyskinesis & $\begin{array}{l}1 \\
5 \\
\text { is }\end{array}$ \\
\hline
\end{tabular}

Figure 4 Meta-analysis results for the studies comparing risk of shoulder pain events between athletes with and without dyskinesis.

which required dichotomisation for the current meta-analysis. A sensitivity analysis excluding this study highlighted homogeneity among the remaining four studies $\left(\mathrm{I}^{2}=17 \%\right){ }^{24343637}$ While the remaining studies found a $28 \%$ increased risk of developing shoulder pain in athletes with scapular dyskinesis, this result was not statistically significant $(\mathrm{RR}=1.28$, CI 0.93 to 1.76$)$. This sensitivity analysis identifies that caution should be taken in interpreting these results and further confirmation studies would be beneficial.

It is possible that the importance of scapular dyskinesis as a risk factor may vary according to age or competitive level. Based on the forest plot (figure 4), there does not appear to be a consistent difference in the effects of dyskinesis within age or competitive level. For example, the results of the two studies performed on adolescent athletes are contrasting. ${ }^{34} 35$ Likewise, the one study performed on recreational athletes has a similar point estimate, although with wider $95 \% \mathrm{CI}$, when compared with the pooled RR. ${ }^{37}$ However, due to the small number of studies overall subgroup analysis is not appropriate. At this point, conclusions cannot yet be made regarding the variance of the importance of scapular dyskinesis on different populations.

Of the four studies excluded at full-text review, only one study prospectively investigated the effect of scapular dyskinesis on injury risk. ${ }^{25}$ That study was excluded because it included both shoulder and elbow injuries. ${ }^{25}$ Of note, the authors found no significant difference in injury risk between those with and without scapular dyskinesis. ${ }^{25}$ This is not surprising as the elbow, being more distant from the scapula than the shoulder, is less likely to be affected by scapular dyskinesis. Additionally, a low shoulder pain incidence of $3.7 \%$ was observed; a much lower incidence than that observed consistently in the current review (range between 18.2\% and 40.3\%), decreasing the power of the study to establish risk factors. It is unlikely that the inclusion of that study in the current review would have affected the overall results of the current analysis.

\section{Mechanisms by which scapular dyskinesis could contribute to the development of shoulder pain}

The association between scapular dyskinesis and shoulder pain has been previously examined by cross-sectional studies, which have been summarised by two conflicting systematic reviews. ${ }^{22} 46$ While the earlier review ${ }^{22}$ demonstrated that SIS was associated with less scapular upward rotation and external rotation, and greater elevation and protraction, the other review ${ }^{46}$ did not. The first study ${ }^{22}$ conducted a meta-analysis, whereas the latter ${ }^{46}$ was a narrative review, which may explain their conflicting conclusions. Neither review was able to determine whether scapular dyskinesis was involved in the mechanism of developing shoulder pain or whether it developed as a consequence of shoulder pain. ${ }^{22}$
However, this current review suggests that asymptomatic athletes with scapular dyskinesis have a higher risk of developing future shoulder pain than those without scapular dyskinesis. It is not yet clear exactly how scapular dyskinesis contributes to shoulder pain and whether scapular dyskinesis may be considered a direct or indirect contributing factor. As a direct factor, previous theories have suggested that scapular dyskinesis could result in subacromial pain syndrome via reduction of the subacromial space, ${ }^{117}$ or that scapular dyskinesis reduces rotator cuff functional strength, thereby increasing the likelihood of the tendon overload with subsequent tendinopathic symptoms. ${ }^{19} 20$ However, scapular dyskinesis may be an indirect interactive risk factor, as recent prospective research has demonstrated that scapular dyskinesis is not a risk factor in isolation but increases risk of shoulder pain the presence of excessive increases in load. ${ }^{47}$ Moreover, this current review demonstrated that dyskinesis can be present in the absence of shoulder pain $165 \%$ of athletes) and shoulder pain can be present in the absence of dyskinesis (25\% of athletes). Thus, scapular dyskinesis may only be important, as an interactive risk factor. Alternatively, it is possible that scapular dyskinesis may not be a risk factor at all, but an early warning indicator of future shoulder pain, acting as the canary in a mineshaft. ${ }^{48}$ Previous research has indicated that immediate (short-term) fatigue ${ }^{49-52}$ and excessive increases in training load, ${ }^{53} 54$ can induce scapular dyskinesis without shoulder pain and separately can also independently induce shoulder pain. Further investigation is warranted to confirm or refute these hypothesis to determine what other factors are involved and how the factors interact to contribute to shoulder pain. However, with the currently available evidence, it appears that scapular dyskinesis acts as an indirect factor that increases the risk of shoulder pain in the presence of other risk factors, but this may change once untested theories are explored.

\section{Screening}

The purpose of investigating risk factors for shoulder pain is twofold; to better understand the mechanisms of shoulder pain and to develop strategies to prevent shoulder pain. ${ }^{55} \mathrm{~A}$ commonly proposed strategy is screening to identify those with a risk factor and intervene with a preventative programme. The question is: should screening for scapular dyskinesis be included as common practice? To answer this question, the prevalence of scapular dyskinesis in asymptomatic athletes and the risk of shoulder pain in athletes without scapular dyskinesis must be taken into account. ${ }^{33}$ Recent evidence suggests a $54 \%$ prevalence of scapular dyskinesis in asymptomatic overhead athletes. ${ }^{23}$ According to the results of the current review, the $46 \%$ of athletes without scapular dyskinesis would be exposed to a $25 \%$ risk, leading to 11 athletes in 100 developing shoulder pain. The $54 \%$ of athletes with scapular dyskinesis would be exposed to 
a 35\% risk (43\% increased risk than those without), leading to 19 athletes in 100 developing shoulder pain. In this instance, 35 athletes would correctly be classified as unlikely and 19 athletes correctly classified as likely to develop shoulder pain. This scenario presents a diagnostic accuracy of 54\%, which is essentially the same as a coin toss. The validity of screening in sports has recently been challenged extensively. ${ }^{556}$ The current review would support these challenges, highlighting that screening for scapular dyskinesis is not a useful approach to predict shoulder pain.

On the other hand, the presence of scapular dyskinesis does indicate an increased risk of developing shoulder pain. The time and financial cost of assessing for scapular dyskinesis is minimal. ${ }^{1}$ The information obtained could be used as part of a battery of tests including other known predictive risk factors such as glenohumeral rotational range, ${ }^{57}$ rotator cuff strength ${ }^{58}$ and previous injury $^{57}$ to determine an individualised injury risk profile. In the screening example above, the positive and negative likelihood ratios of 1.26 and 0.73 , respectively, can be calculated. ${ }^{59}$ These figures indicate that shoulder pain is 1.26 times more likely in athletes who have scapular dyskinesis than those that do not have scapular dyskinesis. They also indicate that shoulder pain is only 0.73 times as likely to occur in people who do not have scapula dyskinesis in comparison to those that do have scapular dyskinesis. Thus, it would seem that screening for shoulder pain, would best be conducted using several predictive risk factors, including scapular dyskinesis.

The presence and extent of scapular dyskinesis has been shown to be influenced by acute and chronic fatigue. ${ }^{50-52} 54$ Scapular dyskinesis is more prevalent directly after a session and typically increases as a season progresses. ${ }^{50-54}$ It is possible that athletes may develop scapular dyskinesis postinitial screening, over the course of a season, and then later develop symptoms, which could explain the number of false negatives. $^{49} 54$ Future studies should consider investigating if serial screening has any superior benefit to baseline screening in isolation.

\section{Injury prevention}

Despite the limitations in screening and targeted preventative interventions, the understanding of risk factors can be useful in injury prevention programmes if implemented on a wider scale. For example, eccentric hamstring weakness has been established as a risk factor for hamstring injuries. ${ }^{6061}$ Rather than specifically targeting only athletes with eccentric weakness, eccentric training exercise interventions applied with high compliance in a non-targeted fashion have been very successful at reducing the incidence of hamstring injuries. ${ }^{62-64}$ The current review demonstrates that scapular dyskinesis may increase the risk of shoulder pain, and this suggests that scapular-focused exercises may be of value for injury prevention. However, as discussed earlier, it remains unclear if scapular dyskinesis is directly or indirectly involved in the mechanism of shoulder pain. Addressing scapular dyskinesis in isolation is unlikely to be effective if the scapular dyskinesis is, for example, an adaptive compensation secondary to excessive training load, whereby loading issues should first be addressed. Moreover, evidence from two recent systematic reviews highlights inconsistencies in outcomes of scapular-focused interventions on scapular positioning. ${ }^{656}$ Thus, it cannot be assumed that addressing scapular dyskinesis directly will reduce injury risk. In these instances, screening scapular dyskinesis in athletes may still be useful to identify those with an increased injury risk where intervention is appropriate, even if the intervention addresses other modifiable factors to 'offset' the risk. Two randomised control trials in swimmers ${ }^{67}$ and handball athletes ${ }^{68}$ have demonstrated that a shoulder injury prevention programme using a combination of rotator cuff strength, scapular stability, kinetic chain mobility and energy transfer exercises can be effective in reducing the incidence of shoulder pain. ${ }^{6768} \mathrm{It}$ is impossible to determine from those studies, if scapular-focused interventions, non-scapular focused interventions or a combination is the most effective. There is always inherent overlap between exercises, and it is impossible to isolate rehabilitation of the scapula without some transfer into the kinetic chain. ${ }^{1}$ Based on current evidence, it appears that clinicians should continue to consider scapular position during holistic shoulder exercises designed to promote upper limb robustness and reduce injury susceptibility.

\section{Strength and limitations}

The main strength of this study was its meta-analysis of over 400 participants and the high prevalence of shoulder pain across the included studies, providing greater power to detect risk factors, than given in individual studies. The all-encompassing definition of shoulder pain and the dichotomisation of scapular dyskinesis improves the external validity of the findings, which can be applied from recreational to elite athletes across a multitude of sports.

On the other hand, wide definitions of shoulder pain limit the extrapolation of this study directly to mechanisms of shoulder pain. Additionally, the quality appraisal identified several factors which allow the potential risk of bias. No study reported on whether participants were blinded to the results of their baseline testing. Informing patients of their testing may cause them to alter behaviours, especially if they feel at risk due to 'impaired scapular control'. This may have in turn affected their injury risk, confounding the results observed. The majority of studies did not adequately compare between groups for confounding factors and thus it cannot be determined if scapular dyskinesis is directly or indirectly involved in the mechanism of shoulder pain development.

\section{Future studies}

It is unclear if scapular dyskinesis is directly or indirectly related to the mechanisms of shoulder pain. Future studies should aim to clarify this relationship. It is also worth investigating if scapular dyskinesis interacts with other risk factors through the calculation and combination of likelihood ratios for different

\section{What do we already know?}

Scapular dyskinesis is common in asymptomatic overhead athletes.

- Scapular dyskinesis is associated with poorer outcome in patients with shoulder pain.

\section{What are the new findings?}

- The presence of scapular dyskinesis in asymptomatic overhead athletes indicated a $43 \%$ increased risk of developing shoulder pain.

- This finding in isolation has minimal use in screening but may be useful as part of a battery of tests that includes other known risk factors. 
risk factors. Finally, future studies should investigate if scapular focused treatment can effectively reduce injury risk. It is imperative that any future study investigating scapular dyskinesis as a risk factor for shoulder pain use prospective study design, blind participants to the results of their baseline assessment, and make clear between-group analysis of potential confounding factors at baseline and at end point.

\section{Conclusion}

The presence of scapular dyskinesis in asymptomatic athletes appears to increase the risk of developing shoulder pain by $43 \%$ (CI). This information may be useful as part of the periodic health examination and in the design of injury prevention programmes.

Acknowledgements The authors would like to acknowledge $\operatorname{Dr}$ B Clarsen and Dr H Shitara for providing further raw data and information that allowed the full completion of this review.

Contributors All five authors were responsible for the conception and design of this review. DH and VS conducted the search strategy, study selection, quality assessment and data extraction. LM acted as the third reviewer in cases of disagreement. DH and VC conducted the meta-analysis. DH and VS were primarily responsible for writing the manuscript. However, all five authors contributed substantially to interpreting findings, reviewing the manuscript and preparing the final submission.

\section{Competing interests None declared.}

Provenance and peer review Not commissioned; externally peer reviewed.

(c) Article author(s) (or their employer(s) unless otherwise stated in the text of the article) 2018. All rights reserved. No commercial use is permitted unless otherwise expressly granted.

\section{REFERENCES}

1 Kibler WB, Ludewig PM, McClure PW, et al. Clinical implications of scapular dyskinesis in shoulder injury: the 2013 consensus statement from the 'Scapular Summit'. Br J Sports Med 2013;47:877-85.

2 Kibler WB, Wilkes T, Sciascia A. Mechanics and pathomechanics in the overhead athlete. Clin Sports Med 2013;32:637-51.

3 McHardy A, Pollard H, Luo K. One-year follow-up study on golf injuries in australian amateur golfers. Am I Sports Med 2007;35:1354-60.

4 Clarsen B, Bahr R, Heymans MW, et al. The prevalence and impact of overuse injuries in five Norwegian sports: application of a new surveillance method. Scand J Med Sci Sports 2015;25:323-30.

5 Myklebust G, Hasslan L, Bahr R, et al. High prevalence of shoulder pain among elite Norwegian female handball players. Scand J Med Sci Sports 2013;23:288-94.

6 Abrams GD, Renstrom PA, Safran MR. Epidemiology of musculoskeletal injury in the tennis player. Br I Sports Med 2012;46:492-8.

7 Wanivenhaus F, Fox AJ, Chaudhury S, et al. Epidemiology of injuries and prevention strategies in competitive swimmers. Sports Health 2012;4:246-51.

8 Fuller CW, Taylor A, Kemp SP, et al. Rugby World Cup 2015: World Rugby injury surveillance study. Br J Sports Med 2017;51.

9 Kelly BT, Barnes RP, Powell JW, et al. Shoulder injuries to quarterbacks in the national football league. Am J Sports Med 2004;32:328-31.

10 Clarsen B. Current severity measures are insufficient for overuse injuries. Science and Medicine in Football 2016:2:91-2.

11 Lewis J. Rotator cuff related shoulder pain: assessment, management and uncertainties. Man Ther 2016;23:57-68.

12 Lewis J, McCreesh K, Roy JS, et al. Rotator cuff tendinopathy: navigating the diagnosis-management conundrum. J Orthop Sports Phys Ther 2015;45:923-37.

13 Lewis JS. Rotator cuff tendinopathy/subacromial impingement syndrome: is it time for a new method of assessment? Br I Sports Med 2009;43:259-64.

14 Chester R, Jerosch-Herold C, Lewis J, et al. Psychological factors are associated with the outcome of physiotherapy for people with shoulder pain: a multicentre longitudinal cohort study. Br J Sports Med 2016:bjsports-2016-096084.

15 Littlewood C, May S, Walters S. Epidemiology of rotator cuff tendinopathy: a systematic review. Shoulder \& Elbow 2013;5:256-65.

16 Cools AM, Michener LA. Shoulder pain: can one label satisfy everyone and everything? Br J Sports Med 2017;51.

17 Silva RT, Hartmann LG, Laurino CF, et al. Clinical and ultrasonographic correlation between scapular dyskinesia and subacromial space measurement among junior elite tennis players. Br I Sports Med 2010;44:407-10.

18 Seitz AL, McClure PW, Lynch SS, et al. Effects of scapular dyskinesis and scapular assistance test on subacromial space during static arm elevation. J Shoulder Elbow Surg 2012;21:631-40.
19 Tate AR, McClure PW, Kareha S, et al. Effect of the scapula reposition test on shoulder impingement symptoms and elevation strength in overhead athletes. J Orthop Sports Phys Ther 2008;38:4-11.

20 Kibler WB, Sciascia A, Dome D. Evaluation of apparent and absolute supraspinatus strength in patients with shoulder injury using the scapular retraction test. Am J Sports Med 2006;34:1643-7.

21 Phadke V, Camargo P, Ludewig P. Scapular and rotator cuff muscle activity during arm elevation: a review of normal function and alterations with shoulder impingement. Rev Bras Fisioter 2009;13:1-9.

22 Timmons MK, Thigpen CA, Seitz AL, et al. Scapular kinematics and subacromialimpingement syndrome: A meta-analysis. J Sport Rehabil 2012;21:354-70.

23 Burn MB, McCulloch PC, Lintner DM, et al. Prevalence of scapular dyskinesis in overhead and nonoverhead athletes: a systematic review. Orthop I Sports Med 2016;4:232596711562760

24 Clarsen B, Bahr R, Andersson SH, et al. Reduced glenohumeral rotation, external rotation weakness and scapular dyskinesis are risk factors for shoulder injuries among elite male handball players: a prospective cohort study. Br I Sports Med 2014;48:1327-33.

25 Myers JB, Oyama S, Hibberd EE. Scapular dysfunction in high school baseball players sustaining throwing-related upper extremity injury: a prospective study. J Shoulder Elbow Surg 2013;22:1154-9.

26 Moher D, Shamseer L, Clarke M, et al. Preferred reporting items for systematic review and meta-analysis protocols (PRISMA-P) 2015 statement. Syst Rev 2015:4:1-9.

27 Endnote [program]. Philadelphia, USA:.

28 Covidence systematic review software. Melbourne, Australia: Innovation VH. www. covidence.org.

29 Downs SH, Black N. The feasibility of creating a checklist for the assessment of the methodological quality both of randomised and non-randomised studies of health care interventions. J Epidemiol Community Health 1998;52:377-84.

30 Cochrane handbook for systematic reviews of interventions. In: Higgins J, Green S, Julian PT, eds. The Cochrane Collaboration. Hoboken, NJ: John Wiley \& Sons, 2011.

31 Whittaker JL, Small C, Maffey L, et al. Risk factors for groin injury in sport: an updated systematic review. Br J Sports Med 2015;49:803-9.

32 The Cochrane Callobration. Covidence [program]. Copenhagen: The Nordic Cochrane Centre. 2014

33 Green S. Cochrane handbook for systematic reviews of interventions version. version 5.1 Cochrane Database Syst Rev. 2011.

34 Shitara H, Kobayashi T, Yamamoto A, et al. Prospective multifactorial analysis of preseason risk factors for shoulder and elbow injuries in high school baseball pitchers. Knee Surg Sports Traumatol Arthrosc 2015.

35 McKenna L, Straker L, Smith A. Can scapular and humeral head position predict shoulder pain in adolescent swimmers and non-swimmers? I Sports Sci 2012:30:1767-76

36 Kawasaki T, Yamakawa J, Kaketa T, et al. Does scapular dyskinesis affect top rugby players during a game season? I Shoulder Elbow Surg 2012;21:709-14.

37 Struyf F, Nijs J, Meeus M, et al. Does scapular positioning predict shoulder pain in recreational overhead Athletes? Int I Sports Med 2014:35:75-82.

38 DeMatas K, Taylor W. Glenohumeral internal rotation deficit: a predictor of shoulder/ elbow injury in professional female tennis players. American Medical Society for Sports Medicine 2015 annual meeting: research poster presentations. Clinical journal of sports medicine 2015:174-209.

39 Laudner KG, Stanek JM, Meister K. Differences in scapular upward rotation between baseball pitchers and position players. Am J Sports Med 2007;35:2091-5.

40 Tyler TF, Mullaney MJ, Mirabella MR, et al. Risk factors for shoulder and elbow injuries in high school baseball pitchers: the role of preseason strength and range of motion. Am J Sports Med 2014:42:1993-9.

41 Kibler WB, Uhl TL, Maddux JW, et al. Qualitative clinical evaluation of scapular dysfunction: a reliability study. J Shoulder Elbow Surg 2002:11:550-6.

42 Uhl TL, Kibler WB, Gecewich B, et al. Evaluation of clinical assessment methods for scapular dyskinesis. Arthroscopy 2009:25:1240-8.

43 McClure P, Tate AR, Kareha S, et al. A clinical method for identifying scapular dyskinesis, part 1: reliability. J Ath/ Train 2009;44:160-4.

44 Tate AR, McClure P, Kareha S, et al. A clinical method for identifying scapular dyskinesis, part 2: validity. J Athl Train 2009;44:165-73.

45 Struyf F, Nijs J, De Coninck K, et al. Clinical assessment of scapular positioning in musicians: an intertester reliability study. J Athl Train 2009;44:519-26.

46 Ratcliffe E, Pickering S, McLean $S$, et al. Is there a relationship between subacromial impingement syndrome and scapular orientation? A systematic review. Br I Sports Med 2014:48:1251-6.

47 Møller M, Nielsen RO, Attermann J, et al. Handball load and shoulder injury rate: a 31 week cohort study of 679 elite youth handball players. Br J Sports Med 2017:51:.

48 Egger G, Dixon J. Non-nutrient causes of low-grade, systemic inflammation: support for a 'canary in the mineshaft' view of obesity in chronic disease. Obes Rev 2011;12:339-45

49 Madsen PH, Bak K, Jensen S, et al. Training induces scapular dyskinesis in pain-free competitive swimmers: a reliability and observational study. Clin I Sport Med 2011;21:109-13. 
50 Joshi M, Thigpen CA, Bunn K, et al. Shoulder external rotation fatigue and scapular muscle activation and kinematics in overhead Athletes. J Ath/ Train 2011;46:349-57.

51 Maenhout A, Dhooge F, Van Herzeele M, et al. Acromiohumeral distance and 3-dimensional scapular position change after overhead muscle fatigue. J Ath/ Train 2015;50:281-8.

52 Rich RL, Struminger AH, Tucker WS, et al. Scapular upward-rotation deficits after acute fatigue in tennis players. J Athl Train 2016;51:474-9.

53 Hibberd EE, Laudner KG, Kucera KL, et al. Effect of swim training on the physical characteristics of competitive adolescent swimmers. Am J Sports Med 2016:44:2813-9.

54 Laudner KG, Lynall R, Meister K. Shoulder adaptations among pitchers and position players over the course of a competitive baseball season. Clin J Sport Med 2013;23:184-9.

55 Bahr R. Why screening tests to predict injury do not work-and probably never will...: a critical review. Br J Sports Med 2016;50:776-80.

56 Whiteley R. 'Moneyball' and time to be honest about preseason screening: it is a sham making no inroads on the 1 billion dollar injury costs in baseball. Br J Sports Med 2016;50.

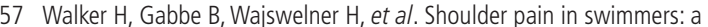
12-month prospective cohort study of incidence and risk factors. Phys Ther Sport 2012;13:243-9.

58 Swanik KA, Lephart SM, Swanik CB, et al. The effects of shoulder plyometric training on proprioception and selected muscle performance characteristics. J Shoulder Elbow Surg 2002;11:579-86.

59 Kent P, Hancock MJ. Interpretation of dichotomous outcomes: sensitivity, specificity, likelihood ratios, and pre-test and post-test probability. J Physiother 2016;62:231-3.
60 Opar DA, Williams MD, Timmins RG, et al. Eccentric hamstring strength and hamstring injury risk in australian footballers. Med Sci Sports Exerc 2015; $47: 857-65$.

61 Timmins RG, Bourne MN, Shield AJ, et al. Short biceps femoris fascicles and eccentric knee flexor weakness increase the risk of hamstring injury in elite football (soccer): a prospective cohort study. Br J Sports Med 2016;50:1524-35.

62 Brukner P. Hamstring injuries: prevention and treatment-an update. Br J Sports Med 2015:49:1241-4

63 Goode AP, Reiman MP, Harris L, et al. Eccentric training for prevention of hamstring injuries may depend on intervention compliance: a systematic review and metaanalysis. Br J Sports Med 2015;49.

64 Al Attar WSA, Soomro N, Sinclair PJ, et al. Effect of injury prevention programs that include the nordic hamstring exercise on hamstring injury rates in soccer players: a systematic review and meta-analysis. Sports Med 2017;47:A35.2-A35.

65 Bury J, West M, Chamorro-Moriana G, et al. Effectiveness of scapula-focused approaches in patients with rotator cuff related shoulder pain: a systematic review and meta-analysis. Man Ther 2016;25:35-42.

66 Reijneveld EA, Noten S, Michener LA, et al. Clinical outcomes of a scapular-focused treatment in patients with subacromial pain syndrome: a systematic review. $\mathrm{Br}$ J Sports Med 2017;51.

67 Swanik KA, Swanik CB, Lephart SM, et al. The Effect of Functional Training on the Incidence of Shoulder Pain and Strength in Intercollegiate Swimmers. J Sport Rehabil 2002;11:140-54.

68 Andersson SH, Bahr R, Clarsen B, et al. Preventing overuse shoulder injuries among throwing Athletes: a cluster-randomised controlled trial in 660 elite handball players. Br J Sports Med 2016 